protruding quite prominently, forming deep valleys at the pseudothreefold axes between the $\mathrm{A}, \mathrm{B}$, and $\mathrm{C}$ subunits. The quasiequivalent $\mathrm{A}, \mathrm{B}$, and $\mathrm{C}$ subunits adopt the jellyroll fold and are very similar in structure. The $N$ termini, which had been found on the outside of the virion immunogenically, are found in the interior of the virion, and the first 26 residues of the A subunit are disordered. The $N$ termini of the $B$ and $C$ subunits, which are completely visible, interact at the interior of the pseudo 6-fold axes, forming annuli. The $\mathrm{C}$ termini are exterior to the virion. All three histidines present in the coat protein are found on the inside of the virion, and may confirm the prediction that these residues bind RNA.

\section{MS04.09.06 STRUCTURE OF HUMAN RHINO VIRUS 50} AT $1.8 \ddot{A}$ E. Blancl, R. S. Alexander', M. McMillan 3 , D. C. Pevear4, V. Giranda5, M. S. ChapmanI, Institute for Molecular Biophysics and Department of Chemistry, Florida State University, Tallahassee, Fl 32306, 2Dupont Merck Pharmaceutical Co., Wilmington, De 19880, 3Eastman Kodak Co., Rochester, NY 14650,4 Viropharma Inc., Malvern, Pa 19335, 5 Abbott Laboratories, Abbott Park, Il 60064-3500

Human Rhino Virus 50 (HRV50) belongs to one of the largest and most important families of viral pathogens: the picomaviridae. Rhinoviruses are among the smallest RNA-containing viruses; their genome is surrounded by a protein shell made of 60 copies of the asymmetric unit, each containing 4 polypeptide chains. As the major cause of the common cold, these viruses have already been extensively studied, and several structures have been determined. HRV 50 is of special interest for two reasons: firstly, it is one of the more representative serotypes, of use in the design of broad spectrum drugs against all rhinoviruses. Secondly, there is the opportunity to study the structure and drug interactions at unusually high resolution, $1.8 \AA$.

The structure refinement has been performed mainly in realspace, using a new technique [Chapman (1995) Acta Crystallogr. A51: 69-80] that accounts for the resolution of the experimental data, and can be 50 times faster than conventional reciprocalspace refinement. The method takes advantage of accurate phases that result from application of 15 -fold non-crystallographic symmetry. Moreover, the method makes it tractable to refine such large structures against all experimental data simultaneously. Prior comparaisons [Chapman \& Rossman (1996) Acta Crystallogr. $D$ : in press] with canine parvovirus have demonstrated that the quality of a real-space refined virus is at least as good as refined in reciprocal space.

MS04.09.07 STRUCTURE-BASED DESIGN OF PEPTIDE PRESENTATION ON A VIRAL SURFACE: THE CRYSTAL STRUCTURE OFA PLANT/ANIMAL VIRUS CHIMERA AT 2.8A RESOLUTION T. Lin 1, C. Porta2, G. Lomonossoff2, J. E. Johnson'1 1Department of Molecular Biology, The Scripps Research Institute, $10666 \mathrm{~N}$. Torrey Pines Rd., La Jolla, CA and 2Department of Virus Research, John Innes Centre, Colney Lane, Norwich NR 4 7UH, UK

A genetically engineered icosahedral plant virus, cowpea mosaic virus (CPMV), was employed as an expression and presentation system to display a 14 amino acid linear antigenic epitope found in a capsid protein of human rhinovirus 14 (HRV14). Gram quantities of the CPMV/HRV14 chimera were made in plants and purified particles were crystallized in a form isomorphous with wild-type CPMV. The $2.8 \AA$ resolution structure of the chimera shows that the inserted loop is well ordered and that, if the loop were intact, a PHE residue of CPMV would be placed in a hydrophilic environment. The resultant strain must make the loop an attractive substrate for endogenous plant proteases, as roughly $80 \%$ of the inserted polypeptides are cleaved, allowing the PHE to be partially buried. Altering the PHE to an ARG should relieve the stress, reducing the propensity for cleavage and increasing the likelihood that the peptide will assume a structure closely similar to its structure in HRV14. Successful crystallization of other CPMV chimeras in forms isomorphous with the native virus suggests that this is a viable system for structure-based design of peptide presentation.

PS04.09.08 STRUCTURAL STUDIES OF AN HRV14:HIV-1 V3 LOOP CHIMERA THAT INDUCES NEUTRALIZING ANTBBODIES AGAINST HIV-1. Edward Annold, Dawn A. Resnick, Jianping Ding, Sheila C. Geisler, Greg Kamer, and Gail Ferstandig Arnold, CABM and Rutgers University Chemistry Department, 679 Hoes Lane, Piscataway, NJ 08854

Human rhinovirus 14 (HRV14) has been engineered for use as a live-virus vaccine against human immunodeficiency virus (HIV-1). An HRV 14:HIV-1 chimeric virus, designated DN-6, contains an immunogenic region from the V3 loop of the gp 120 surface glycoprotein of HIV-1 transplanted into the neutralizing immunogenic site II of HRV14. DN-6 is strongly neutralized by anti-HIV-1 antibody preparations and elicits the production of antisera that potently neutralize HIV-1 in cell culture (Resnick et al., 1995). The crystal structure of DN-6 will reveal the conformation of a V3 loop epitope that is able to elicit a neutralizing response against HIV-1.

Crystals of DN-6 were obtained that diffract X-rays to $2.8 \AA$ resolution. A native dataset measured at CHESS from cooled crystals is $44.8 \%$ complete to $3.5 \AA$ resolution $(238,364$ independent reflections, $R_{\text {merge }}=11.7 \%$ ) and from a flash-frozen crystal is $61.4 \%$ complete to $3.5 \AA$ A resolution $(322,463$ independent reflections, $R_{\text {merge }}=14.7 \%$ ). The crystal symmetry and unit cell dimensions are similar to those of the orthorhombic crystal forms of HRV14 (Erickson et al., 1983) and poliovirus type 1 Mahoney (Hogle et al., 1985). Surprisingly, although the cell dimensions are very similar, the packing is different from that of the poliovirus 1 Mahoney crystals and the space group is P22 $22_{1}$ instead of $\mathrm{P}_{212} 12$. The reflections were indexed accordingly for the standard space group choice $\mathrm{P} 22_{1} 2{ }_{1}$ with $\mathrm{a}=378.4, \mathrm{~b}=354.7$, and $\mathrm{c}=317.7 \AA$.

Rotation and translation functions indicated a particle rotation of $93.0^{\circ}$ (cooled) or $94.2^{\circ}$ (frozen) around the $z$ axis relative to a standard icosahedral orientation and the particle center position to be close to $z=0.25$. Rigid body refinement using the HRV14 native coordinates (Rossmann et al, 1985; Arnold \& Rossmann, 1988) yielded an R-factor of 0.34 for $15-3.5 \AA$ resolution for the cooled dataset. Phase improvement by 30 -fold noncrystallographic symmetry averaging is underway. Electron density is well ordered for the majority of the protein shell. We are in the process of interpreting the density in the chimeric region in terms of the structure of the transplanted HIV-1 V3 loop immunogen.

PS04.09.09 STRUCTURE OF COXSACKIEVIRUS B1 COMPLEXED WITH AN ANTIVIRAL AGENT. Karen N. Lentz ${ }^{1}$, Allen D. Smith1, Sheila C. Geisler ${ }^{1}$, Stu Cox², Jason DeMartino2, V. Girijavallabhan2, John O'Connell2, and Edward Annold 1, 1Center for Advanced Biotechnology and Medicine, and Rutgers University Department of Chemistry, 679 Hoes Lane, Piscataway, NJ 08854, 2 Antiviral Chemotherapy, Schering-Plough Research Institute, 2015 Galloping Hill Road, Kenilworth, NJ 07033

Coxsackievirus B1 (CVB1) is a member of the picornavirus family and causes a wide variety of human diseases. These include common colds, mild febrile illness, encephalitis, and acute myocarditis. The picornaviruses are small (approximately $300 \AA$ 
in diameter) RNA viruses that have an iscosahedrally systematic caspid. This caspid contains 60 copies of each of four coat proteins VP1, VP2, VP3, and VP4. Several structures of picornaviruses have been determined to date, however, no three-dimensional structure is currently available for CVB1. We report here the crystallization, $\mathrm{X}$-ray diffraction analysis, and structure determination of CVB1 complexed to a potent antiviral agent.

Crystals of CVB 1 complexed to an antiviral agent in the SCH 47802 series were grown in the presence of $\mathrm{Li}_{2} \mathrm{SO}_{4}$ and $\mathrm{PEG}$ using a modification of the vapor diffusion technique. Small triangular blocks appear in $2-5$ days, the largest measuring $0.2 \times 0.2 \times 0.3$ $\mathrm{mm}$. These crystals diffract $X$-rays to at least $2.6 \AA$ resolution at CHESS. Data analysis indicates that these crystals belong to space group $\mathrm{C} 2222_{1}$ with cell dimensions of $a=345.7, b=497.2 .4$, and $c=485.9 \AA$, and $\alpha=\beta=\gamma=90^{\circ}$. A dataset was collected at the CHESS Fl station that is $50 \%$ complete to $3.0 \AA$ resolution with a $R_{\text {merge }}$ of $10.6 \%$.

Molecular replacement was used to solve the structure using a starting model of coxsackievirus B3. Rotation and translation functions indicate that the particle is rotated $54.8^{\circ}$ around the $y$ axis relative to a standard icosahedral orientation and that the position of the particle center is at $y=0.19$. After the molecular replacement solution was determined, thirty-fold noncrystallographic symmetry averaging was used to improve the electron density and the phases. Electron density consistent with bound antiviral agent is present in the drug binding pocket. Details of the interactions between the antiviral agent and the virus will be presented, as well as a comparison between CVB1 and other picornaviruses.

PS04.09.10 CRYSTALLIZATION OF EIAV p26. Zhongmin Jin1,3, Ashley J. Birkett2, Ling Jin2, Darrell L. Peterson², Catherine L. Lawson'. 1Biology Department, Brookhaven National Laboratory,Upton, NY11973; 2Department of Biochemistry and Molecular Biophysics, Virginia Commonwealth University, Richmond, VA 23298; 3Department of Physics, State University of New York at Stony Brook, Stony Brook, NY11790, USA

We report here the crystallization of the $\mathrm{p} 26$ core protein from equine infectious anaemia virus (EIAV), a retrovirus of the Lentiviridae family. EIAV is responsible for causing a chronic, debilitating disease in horses. Infection has been reported worldwide and EIAV is recognized as a livestock pathogen of significent economic importance to the horse industry. There is significent homology between the non-human lentiviruses and HIV-1. The structural study of EIAV gag/core protein will help in understanding the structure of HIV core protein, and in evaluating methods of effective treatment and control of viral infection.

Crystals were grown at room temperature by vapor diffusion with $0.1 \mathrm{M}$ Citrate buffer and $10 \%$ PEG3300, $15 \%$ isopropanol, at $\mathrm{pH}$ 6.5. They belong to the space group $\mathrm{P}_{1} 22$ (or P6522) with $a=b=101 \AA$ and $c=158 \AA$. A complete native data set to $3.6 \AA$ $\left(\mathrm{R}_{\mathrm{sym}}=11 \%\right)$ has been collected at beamline $\mathrm{X} 12 \mathrm{C}$ of National Synchrotron Light Source at Brookhaven National Laboratory. We expect to obtain a higher resolution native, since diffraction from one crystal frozen in liquid propane was observed to $2.8 \AA$.

The crystal may contain one or two p26 protomers per asymmetric unit. The space group symmetry and cell dimensions suggest that the packing of $\mathrm{p} 26$ protomers may be similar to packing of HIV-1 p24 protein in $100 \mathrm{~A}$ diameter fibers (Ehrich et al,1992). EIAV p26 and HIV-1 p24 have 55\% sequence homology and $30 \%$ sequence identity.
PS04.09.11 INTERACTION OF INTERCELLULAR ADHESION MOLECULE-I (ICAM-1) AND HUMAN RHINOVIRUS (HRV) AND THE ROLE OF CHARGE. Prasanna R. Kolatkar, Jordi Bella, Wai-ming Lee", Roland Rueckert" and Michael G. Rossmann, Department of Biological Sciences, Purdue University, W. Lafayette, IN 47906 "Institute of Molecular Virology, University of Wisconsin, Madison, WI 53706.

Intercellular Adhesion Molecule-1 (ICAM-1) is the receptor used by the majority of human rhinoviruses (HRVs). We have previously reported a cryo-electron microscopy reconstruction of the ICAM-1:HRV complex (PNAS, 1992) which shows the general features of the binding. Well-diffracting crystals of ICAM-1 expressed in bacullovirus are now being used for crystallographic studies. Nevertheless the crystals have somewhat variable cell constants which change by as much as $10 \AA$ and make MIR phasing problematic. The variability in cell constants is likely attributed to the significant amount of glycosylation. We (Chris Marlor, Jeff Greve; Bayer Inc., W. Haven, CT 06516) have introduced one selenomethionine into ICAM-1 to allow MAD phasing.In addition we are using MAD data collection at the absorption edges of heavy atom derivatives to obtain phase information. We will report the progress of the structure determination of ICAM-1 and its relevance to understanding virus-receptor binding interactions.

The charge surface potentials of several human rhinoviruses have been calculated using X-ray coordinates of HRVs. We have employed site-directed mutagenesis of certain charged residues within the canyon and which overlap with the ICAM-1 footprint determined from the EM reconstruction. The results are consistent with the observed HRV:ICAM-1 interactions.

PS04.09.12 STRUCTURAL STUDIES OF THE ROUS SARCOMA VIRUS (RSV) CAPSID PROTEIN. Ladislau C. Kovaril, Cory Momany 1, Faith Miyagi 1, Rui Zhaol, Stephen Campbell2, Bao Vong2, Volker M. Vogt2, Michael G. Rossmann1, IDepartment of Biological Sciences, Purdue University, West Lafayette, IN 47907, USA, 2Section of Biochemistry, Molecular and Cell Biology, Cornell University, Ithaca, NY 14853, USA

The RSV and HIV virus capsid (CA) proteins exhibit structural and functional similarities. Secondary structural predictions suggest that the two capsid proteins share the same fold. Knowledge of the CA protein structure should prove useful in designing anti-retroviral agents that inhibit viral uncoating, assembly, maturation or release.

Crystals of RSV CA diffract X-rays to $3.5 \AA$ resolution. The crystals belong to the monoclinic space group $C 2$ with unit cell parameters $\mathrm{a}=374.4 \AA, \mathrm{b}=128.1 \AA, \mathrm{c}=200.2 \AA$ and $\beta=121.8^{\circ}$. An asymmetric unit of the crystal should contain 20 to 30 molecules based on reasonable $\mathrm{V}_{\mathrm{M}}$ values. Diffraction data of native and heavy atom derivatives were collected on frozen crystals at home and at CHESS. Self rotation functions suggest that RSV CA crystallizes as a helical array. 